\title{
Editorial for application-driven knowledge acquisition
}

\author{
Xue $\mathrm{Li}^{1} \cdot$ Sen Wang ${ }^{1,2} \cdot$ Bohan $\mathrm{Li}^{3}$ \\ Published online: 25 June 2020 \\ (C) Springer Science+Business Media, LLC, part of Springer Nature 2020
}

This special issue has 8 papers focused on innovative Application-Driven Knowledge Acquisition approaches to problems that involve big data, artificial intelligence, and machine learning applications.

In the era of intelligence, the burgeoning topics of machine learning and knowledge acquisition have changed the traditional way of knowledge acquisition. Information mining based on big data has brought about tremendous changes in many fields. Like deep learning has attracted wide attention in recent years, which has been effectively applied in many fields. In this background, most researches are driven by specific applications and further integrated deep learning models with big data. As a powerful tool, it helps researchers to better mine valuable information from high-dimensional massive data to acquire knowledge more effectively. Following an open call for papers, the articles in this special issue focus on various deep learning models including recurrent neural network (RNN), long and short time memory network (LSTM), bidirectional long and short time memory network (Bi-LSTM), dynamic Bayesian network (DBN) and graph convolution network $(\mathrm{GCN})$, and conduct research on multiple tasks such as knowledge acquisition, knowledge representation and data mining. The special issue received 14 paper (including recommended papers from the 14th-International Conference on Advanced Data Mining and Applications, ADMA2018, Nanjing, China). The acceptance ratio is about $28.5 \%$.

The main ideas of these papers are as follows:

As the popularization of mobile smart devices, the acquisition of knowledge can hardly be separated from essential privacy protection. Aiming at the mobile Internet application scenario,

\section{Xue Li}

xueli@itee.uq.edu.au

Sen Wang

sen.wang@uq.edu.au

Bohan Li

bhli@nuaa.edu.cn

1 The University of Queensland, Brisbane, QLD 4072, Australia

2 Griffith University, Brisbane, Australia

3 Nanjing University of Aeronautics and Astronautics, Nanjing 211106 Jiangsu, China 
J Jiang, S Ji and G Long proposed a novel attention-augmented and decentralized knowledge acquisition framework based on federated learning to achieve decentralization and protect private data.

$\mathrm{T} \mathrm{Wu}, \mathrm{H}$ Wang and their colleagues proposed a complete general technical framework for constructing knowledge graph from multiple online encyclopedias, including knowledge rule extraction, live knowledge extraction, lightweight entity link and semi-supervised entity link, and gives all the technical details.

W Yuan, K He and their colleagues proposed a multi-view network embedding method based on node similarity ensemble. Embedding results can be further leveraged in deep learning frameworks and provide excellent support for downstream tasks.

Medical data analysis based on deep learning has been widely studied in recent years. The article "Deep Learning for Heterogeneous Medical Data Analysis", by L Yue, D Tian and their colleagues, undertakes a theoretical analysis on deep learning model used in medical data mining, and describes a pipeline from application problem, to data structure, applicable model, and evaluation method. They discussed the scope, characteristics, and essential structure of heterogeneous medical data, and summarized the main deep learning models and main tasks involved in analysis of medical data.

Y Wang, W Chen and their colleagues detailed the use of DBN to obtain correlations between medical variables from electronic health records (EHRs) and measure fine-grained patient similarity using RNN. Their approach provides better support for patient treatment and is interpretable.

W Chen, G Long and their colleagues considered the mining of valid information in medical data as well. In order to solve the problem that ISP models rarely consider timeseries and the interaction between multi-organ systems simultaneously, they proposed the AMRNN method, which is a deep interpretable learning model based on the attention mechanism of multi-task network.

In addition to medical field, deep learning models are also widely used in social networks. In "Fine-Grained Emotion Classification of Chinese Microblogs Based on Graph Convolution Networks", Y Lai, L Zhang and their colleagues utilized LSTM to extract the features of words in the sentences, and proposed GCN model based on the syntax parses to capture the complex language grammatical structures of Chinese. Experiments have proved that the use of syntax information can achieve better Fine-Grained Emotion Classification of Chinese Microblogs.

Finally, D Xiao, L Song and their colleagues presented a novel anomalous trajectory detection method named ATD-RNN, which uses RNN to learn trajectory sequence information embedding and take different sources and destinations into consideration to alleviate the data sparsity problem.

Collectively, these papers illustrate the diverse approaches to utilize deep learning model and big data to solve problems, such as sentiment analysis, knowledge graph construction, medical data analysis, decentralized knowledge acquisition, and trajectory detection. It turns out that in these tasks, the models based on deep learning are widely applied in specific areas and achieve state-of-the-art performance, bringing unprecedented welfare and convenience to people's lives. However, the deep learning model is not a 
panacea, and it still has many limitations and urgent problems to be solved. We look forward to more researchers taking up these opportunities and challenges brought with big data applications.

Acknowledgements We thank the reviewers for their useful comments on the papers submitted to this Issue. We would also like to thank the participants and attendees of the ADMA conference for the enlightening presentations and discussions.

Publisher's note Springer Nature remains neutral with regard to jurisdictional claims in published maps and institutional affiliations. 